Jpn. J. Genet. (1995) 70, pp. 675-685

\title{
Mitochondrial plasmid-like DNAs of the B1 family in the genus Oryza: sequence heterogeneity and evolution
}

\author{
Shin-ichi Miyata ${ }^{1}$, Akira Kanazawa ${ }^{1}$, Nobuhiro Tsutsumi ${ }^{1}$, Yoshio Sano ${ }^{2}$ \\ and Atsushi HiraI ${ }^{1, *}$ \\ ${ }^{1}$ Laboratory of Radiation Genetics, Faculty of Agriculture, The University \\ of Tokyo, Yayoi 1-1-1, Bunkyo-ku, Tokyo 113, \\ ${ }^{2}$ Plant Breeding Institute, Faculty of Agriculture, Hokkaido \\ University, Kita 9, Nishi 9, Sapporo 060, Japan
}

(Received 28 August 1995)

\begin{abstract}
Four kinds of circular plasmid-like DNA, designated B1, B2, B3 and B4, have been found in the mitochondria of Oryza sativa L. with an AA genome. Three novel B1-homologous mitochondrial plasmid-like DNAs, designated, M1, M2 and M3, were isolated in the present study from strains with CC and CCDD genomes in the genus Oryza. We cloned and sequenced these DNAs and found that the sequences of these molecules have wide regions of homology. B1, M2 and M3 each lack about $300 \mathrm{bp}$ of a region that is present in M1 and small repeats were found at the sites of deleted sequences. Therefore, we propose the hypothesis that the B1 family differentiated from a common ancient molecule that was similar to M1 via, probably, slipped mispairing during DNA replication at several stages in the evolution in the genus Oryza.
\end{abstract}

\section{INTRODUCTION}

The mitochondria of some higher plants contain one or more kinds of small circular or linear DNA molecule in addition to the main genome (for review, see Pring and Lonstale, 1985). These small DNAs are referred to as plasmid-like DNAs.

Four kinds of circular plasmid-like DNA were originally detected in the mitochondria of cultivated rice (Oryza sativa L.) and they were named B1, B2, B3 and B4. They have been cloned and their nucleotide sequences have been determined (Yamaguchi and Kakiuchi, 1983; Shikanai et al., 1987, 1989; Shikanai and Yamada, 1988). B1, B2, B3 and B4 are 2,135, 1,546, 1,514 and $969 \mathrm{bp}$ in length, respectively. Most of the known circular plasmid-like DNAs exhibit no obvious homology to the main mitochondrial genome (Chase and Pring, 1985, 1986; Kemble and Bedbrook, 1980). In O. sativa, the four kinds of plasmid-like DNA also exhibit no obvious homology to the main mitochondrial genome. Therefore,

* Corresponding author. 
it appears that the circular plasmid-like DNAs must be transmitted to progeny independently of the main mitochondrial genome.

Several kinds of homologous plasmid-like DNA have been detected in the mitochondria of sugarbeet (Thomas, 1986) and Vicia fava (Wahleithner and Wolstenholme, 1987; Flamand et al., 1993) and the mechanisms of their generation have been explained simply in terms of recombination or slipped mispairing during DNA replication. In O. sativa, no extensive homology exists among the four kinds of plasmid-like DNA and, therefore, the origin or the mechanism of generation of $\mathrm{B} 1, \mathrm{~B} 2, \mathrm{~B} 3$ and $\mathrm{B} 4$ remains elusive.

The four kinds of plasmid-like DNA were found in many strains of $O$. sativa and wild species with the AA genome in the genus Oryza, and their distribution was investigated (Kanazawa et al., 1992; Miyata et al., 1995). However, in strains in the genus Oryza with $\mathrm{BB}, \mathrm{BBCC}, \mathrm{CC}, \mathrm{CCDD}$ and $\mathrm{EE}$ genomes, no plasmid-like DNA identical to B1, B2, B3 or B4 has been detected with the exception of B1and $\mathrm{B} 2$-homologous molecules in the strains with $\mathrm{CC}$ and CCDD genomes. We designated these B1- and B2-homologous DNA as B1 and B2 family of plasmid-like DNA, respectively, because they were different from B1 and B2 in their molecular sizes and restriction maps (Miyata et al., 1995).

Here we report the identification and characterization of three novel plasmidlike DNAs of the B1 family, which we designated M1, M2 and M3. These DNAs were isolated from mitochondria of strains in the genus Oryza with CC and CCDD genomes and we present their complete nucleotide sequences. From a comparison of the sequences of B1, M1, M2 and M3, we present a hypothesis about the generation of the B1 family. Furthermore, the relationships between these DNA molecules and the diversification of the genus Oryza is discussed.

\section{MATERIALS AND METHODS}

Plant materials and the preparation of total and mitochondrial DNA

Seven perennial strains in the genus Oryza with CC and CCDD genomes, as listed in Table 1, were obtained from the National Institute of Genetics, Japan. These strains were maintained as plants. O. sativa cv. Chinsurah Boro II, which has four kinds of plasmid-like DNAs, was used as the standard. Total DNA was extracted from mature leaves of each strain by the method of Honda et al. (1990). As for O. officinalis W0065, sufficient amount of material was obtained, thus mitochondrial DNA was isolated from mature leaves by the method of Iwahashi et al. (1992).

\section{Southern hybridization with a non-radioactive probe}

Total DNA and mitochondrial DNA were digested with various restriction endonucleases and Southern hybridization analysis was performed as described previously (Iwahashi et al., 1992). Cloned B1 DNA (Sakamoto et al., 1989) was 
Table 1. Strains examined and the plasmid-like DNAs detected in this study

\begin{tabular}{clcc}
\hline \hline Genome & \multicolumn{1}{c}{ Species } & Strain & B1 family \\
\hline AA & O. sativa & Chinsurah Boro II & B1 \\
\hline CC & O. officinalis & W0012 & M1 \\
& & W0564 & M1 \\
& & W0065 & M2 \\
\hline CCDD & O. latifolia & W0542 & M3 \\
& & W1175 & M3 \\
& O. alta & W0017 & M3 \\
& O. grandiglumis & W1194 & M3 \\
\hline
\end{tabular}

labeled with a DIG DNA labeling and detection kit (Boehringer Mannheim, Germany) and used for the probe.

\section{Cloning and sequencing of $M 1, M 2$ and $M 3$}

M1, M2 and M3 were cloned into the $\lambda$ gt11 vector with a Cloning kit (Stratagene, USA) and were then sub-cloned into pBluescript II (Stratagene). Total DNA from O. officinalis W0564 and O. latifolia W0542 was used for the cloning of M1 and M3, respectively. Mitochondrial DNA of $O$. officinalis W0065 was used for the cloning of M2. Each DNA was digested first with EcoRI, then fractions of DNA containing fragments of M1, M2 and M3 were recovered from agarose gels after electrophoresis and cloned into the EcoRI site of $\lambda$ gt11. B1 was used as the probe for screening the clones from each phage clone bank. M1 and M2 were digested into large and small fragments by treatment with $E c o$ RI, respectively, for cloning. Nucleotide sequences were determined with a DNA sequencer (Perkin Elmer ABD, USA).

\section{Polymerase chain reaction (PCR)}

The synthesis of oligonucleotides and PCR were performed with a DNA synthesizer (Perkin Elmer ABD) and a DNA Amplification system (Perkin Elmer Cetus, USA), respectively. The sequences of the primers were as follows:

primer 1, 5'-AGTCACATTTAATGGCTAGA-3';

primer 2, 5'GTCAACTAGTTTATTGAATG-3';

primer 3, 5'-CATACCGGACAGAAATACAG-3'.

The locations and orientations of primers are indicated by small arrows in Figure 4. Primers were designed for amplification of the partial sequences of M1, M2 and M3, which contained EcoRI sites. For each amplification, $250 \mathrm{ng}$ of primers and $100 \mathrm{ng}$ of template DNA were added to the reaction mixture. The conditions for denaturation, annealing and extension were 1 min., 2 min. and 2 min., at $94^{\circ} \mathrm{C}$, $45^{\circ} \mathrm{C}$ and $72^{\circ} \mathrm{C}$, respectively. Amplified fragments of DNA were cloned into 
pBluescript and sequenced.

\section{RESULTS}

Detection of members of the B1 family in the strains with CC and CCDD genomes

We performed Southern hybridization with the B1 probe using total DNA from the strains with CC and CCDD genomes, as listed in Table 1, and we detected B1-homologous plasmid-like DNAs that we named members of the B1 family (Miyata et al., 1995). Chinsurah Boro II was used as the standard strain because its mitochondria contains four kinds of plasmid-like DNA. When total DNA was treated with only RNase A, circular mitochondrial plasmid-like DNAs that hybridized to B1 were detected (in lanes 1 and 2 in Fig. 1) as the signals that corresponded to open circular (oc) and covalently closed circular (cce) forms. As shown in lane 3 in Figure 1, B1 was linearized by EcoRI and converted into a single signal of $2.1 \mathrm{~kb}$. In lane 4, two bands, corresponding to $1.5 \mathrm{~kb}$ and $0.9 \mathrm{~kb}$, were detected in the case of $O$. officinalis W0564. Therefore, we deduced that this molecule was a circular plasmid-like DNA with homology to B1, and we named it M1. M1 was detected in O. officinalis W0564 and W0012, each of which has a CC genome. When total DNA of $O$. officinalis W0065, with a CC genome, was

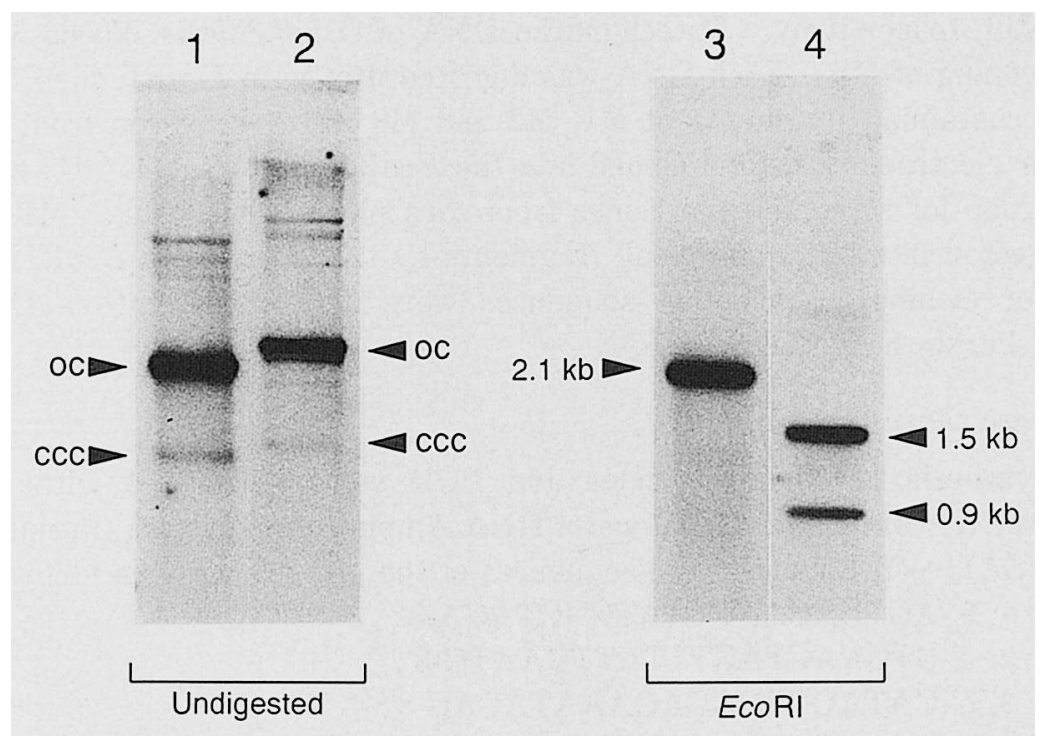

Fig. 1. Southern hybridization of mitochondrial plasmid-like DNA B1 to total DNA. Total DNA was subjected to electrophoresis, transferred to a filter and allowed to hybridize with digoxigenin-labeled B1. Lanes 1 and 3, O. sativa cv. Chinsurah Boro II; lanes 2 and 4, O. officinalis W0564. Samples of total DNA were not (lane 1 and 2) and were treated (lane 3 and 4) with EcoRI. The positions of the open circular (oc) and the covalently closed circular (ccc) forms of DNA are indicated on the both sides of lanes 1 and 2. 
treated with $E c o$ RI, the B1 probe hybridized to fragments of $1.1 \mathrm{~kb}$ and $0.9 \mathrm{~kb}$ (data not shown). We named this circular DNA M2. In the case of O. latiforia W0542, one fragment of $2.1 \mathrm{~kb}$ was detected. This fragment gave a different restriction pattern from that of $\mathrm{B} 1$ when it was treated with other several restriction enzymes, so we named it M3 (data not shown). M3 was detected in four strains of three species with a CCDD genome (Table 1).

\section{Nucleotide sequences and properties of $M 1$, M2 and $M 3$}

We cloned M1, M2 and M3 into a phage vector with an EcoRI site (see MATERIALS AND METHODS). If there had been some EcoRI sites close together, small $E c o$ RI fragments would not have been detected and cloned by the present method. In order to determine whether any closely adjacent $E c o$ RI sites were present, we amplified the partial sequences of M1, M2 and M3 that contained $E c o$ RI sites by PCR, and then we cloned and sequenced the amplified fragments. The complete nucleotide sequences of M1 (2,430 bp), M2 (2,034 bp) and M3 (2,096 $\mathrm{bp}$ ) were determined and alignment of the nucleotide sequences of M1, M2, M3 and $\mathrm{B} 1$ is shown in Figure 2. From comparisons of sequences, we found that the $\mathrm{B} 1$ family also exhibits homology to $\mathrm{B} 2$ and $\mathrm{B} 3$ of rice and to a 1.9-kb plasmid-like DNA of Black Mexican Sweet maize, as indicated by several open boxes. The presence of these homologous regions was reported previously (Shikanai et al., 1989). We also identified a new region of M1 with homology to B2 (as indicated by a1 in Fig. 2).

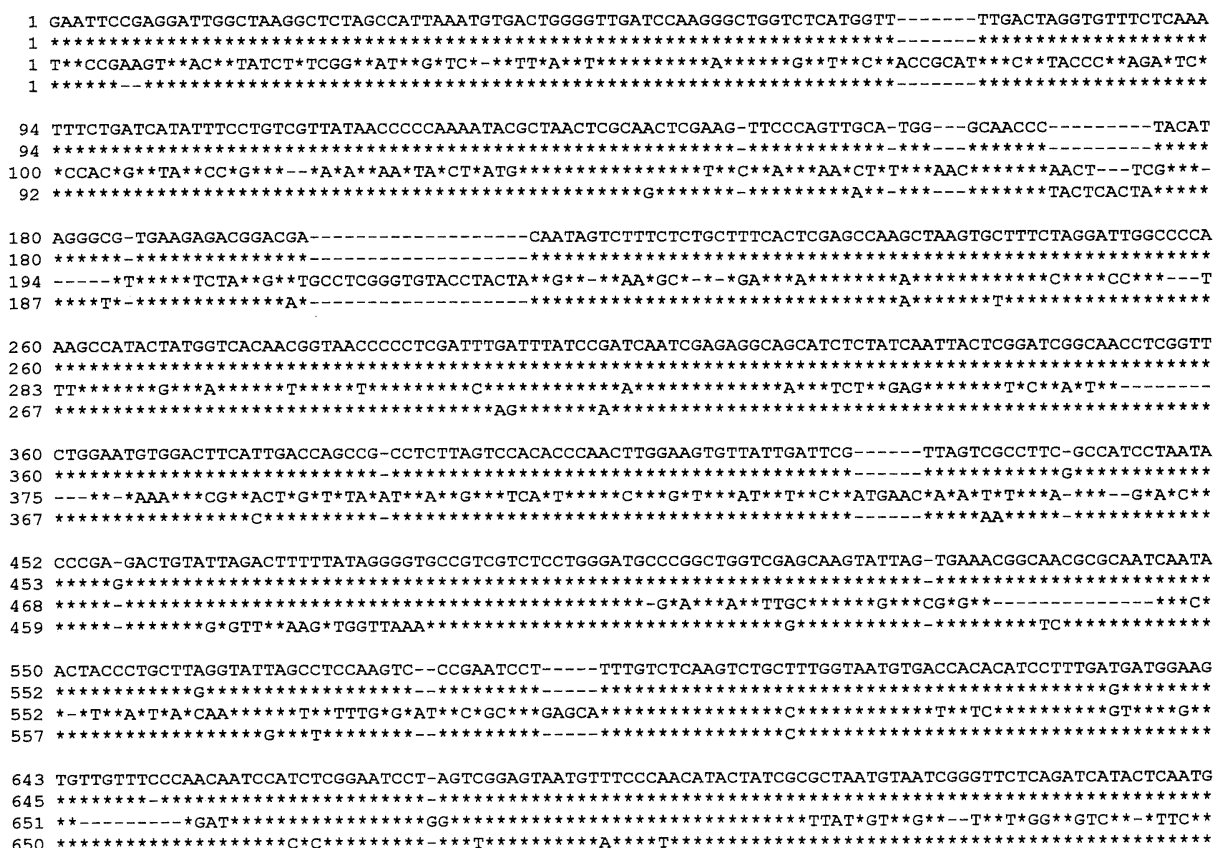


742 AGGGGTGGCTAGTATCCCGAA----ACTTTG-AAG--AACATGAGTTAAGTAGTTCATTCACTTGGTTTCGCCACCAGTCAAAACGCCCTAAAACCCAGT

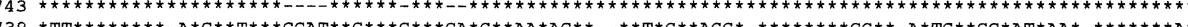

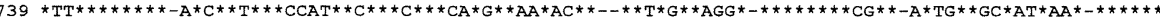

b1

835 AGGTTT CTCGATCACCATTCTACGATTTTATTTCCACCAATTGATTGGTGTTGAAPCATAAAAGTGTGGATCATTATAATAATGGTGTTATTATTACC

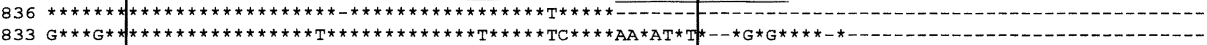

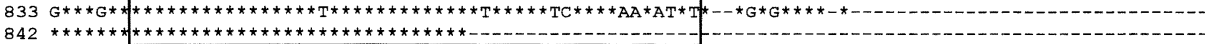

935 TATTAAAAGTGGAAATTAGCCCCTTTAGCACTTCTAAACCTGGTTGTATGCGTTCATTTGAGGGATAGAACTATTGTTTGGTATACCCTAATGGTAGTTT -

a1

M1 1035 CAATCTAAAGTGTTGAACTTGCGTTGG PAGCGCATTTCATTTAAGAAAACAACTCCTGCTCAGATGCGTCTATTTCTCAGGTTTGAGGTAGTCCCTTTAT

M3

B1

$$
878
$$

1235 TTATCACTAAAATCAATAAGTTTATGAATGCAC GAAAGTTGCCTCATAAA-AAGTAGATGTTAGTACCC

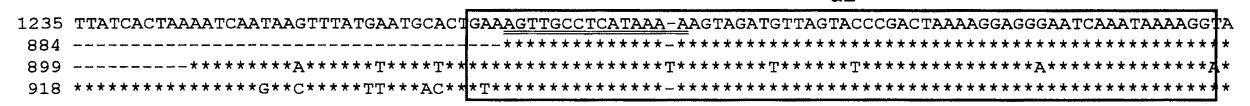

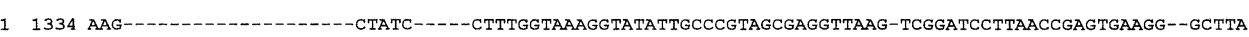
1334 AAG- 946 -

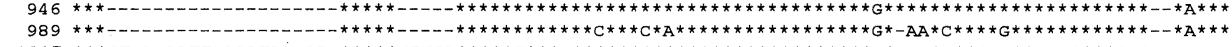

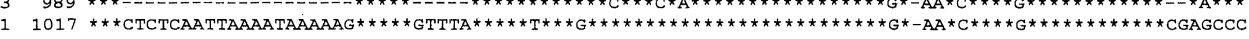

M1 1407 GgTAAAGCACTAAAGTAAAAGCACTAAAGTAGTGG-----TTCTACTTCATTAGCTATCAAGTGTTTGTTGTATCAATTACTTTATTGTGTCAACTAGTT

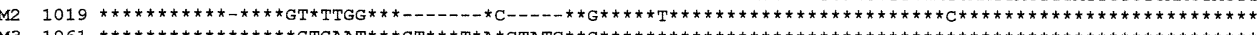

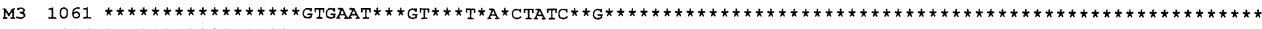

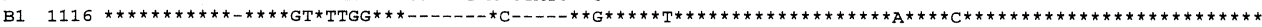

M1 1502 TATTGAATGAAAAgGAATTCCCTCGATAATGGATTTCACTTTTAGGAAAGATAATTAGTTATCCTCCCTCCCTCGAACTGAGCTATTTCCTGTGTATTG

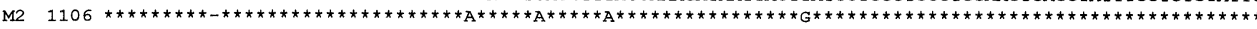

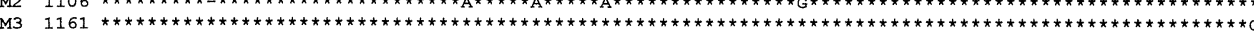

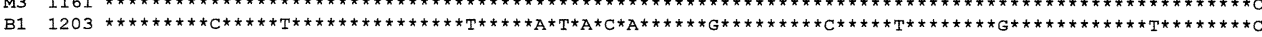

\section{C1}

M1 1602 AATTGTATACACGAGGCTGTCTT TCCTCTGCTCAATCTCCCGACAGGTATGAAATCATAAT CTTT--GGTTTTGTTGTCAATGTAGTGAGCGGA

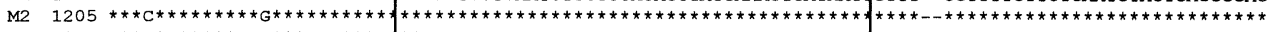

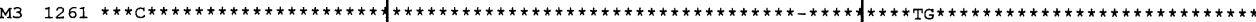

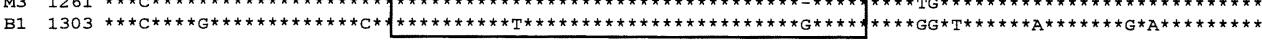

M1 1700 GGAGATACATCCTTCCATCC-AAAGG-GTGTATGTCCCGTAGCGAGTGGAATGGCTCCT-CCGGTTTAGTCGTATAGAACGGGTTGAGTTCTTTTTATGG

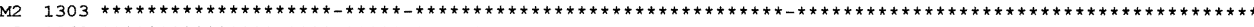

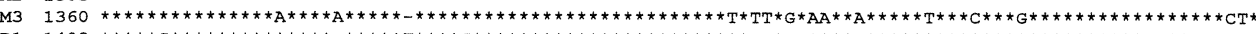

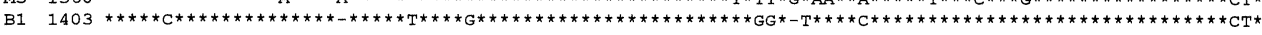

M1 1797 AGTCCTTTCGAGGCTGGAAGTGTTTCTTACAGTTTCCTTTTCCGAAAGGGAAGTCAAGTAAAGGGTTAAAGGCTAGTTAATTC-TCTTTAAGTTTTTCC

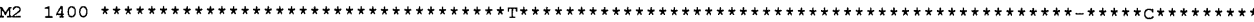

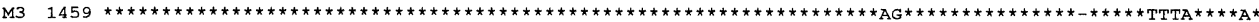

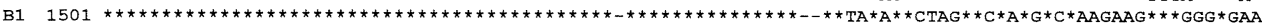

M1 1896 AAGCGAAGAAAG-ACTGTTCCTTAAAAAGGTAAGATAGTTCAGTCCAGTCTTTCGAAGCGTTAGTAGGTGCTCGGAAGGGGTTGGGGATTCCAAAGGGGA

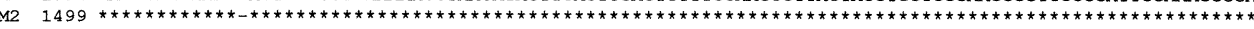

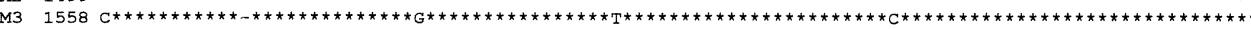

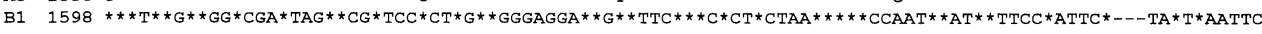

M1 1898 AGGGGAAACCGCACCCCTTTGTTCAATCAACAATTTAAGTAAAAAAACTCGACTTTTCTATTCCGTGCCATTCCTTTCTGTTCCGTTCATCCAACATTG-

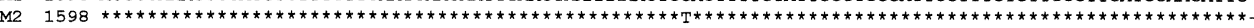

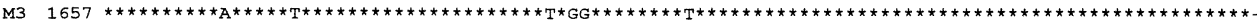

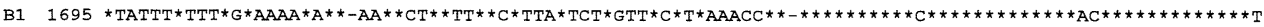

c2

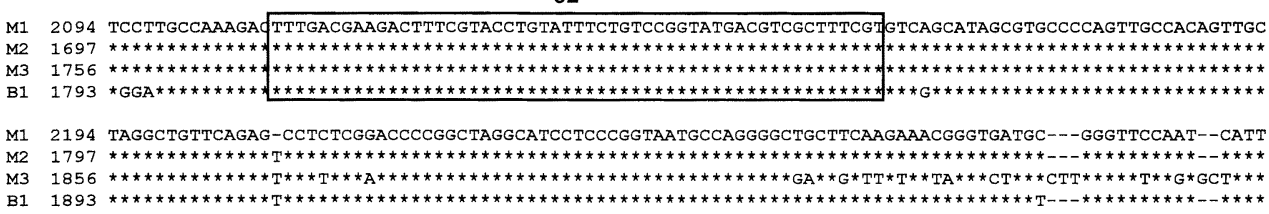




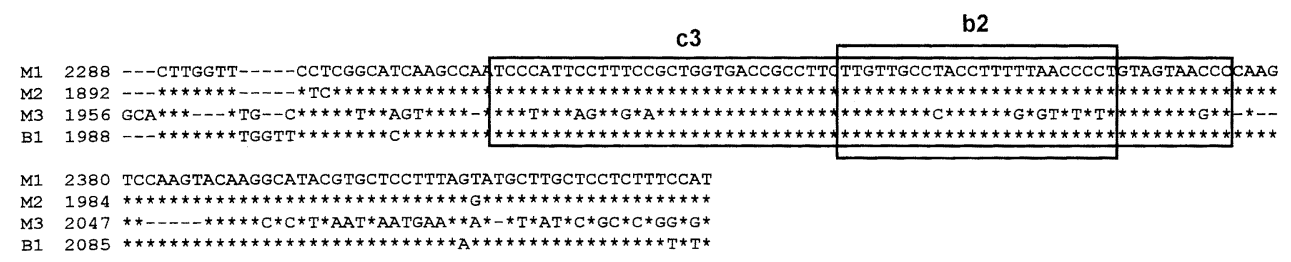

Fig. 2. Alignment of the complete nucleotide sequences of M1, M2, M3 and B1. The nucleotides that are the same as those in M1 are indicated by asterisks and several gaps, introduced to maximize alignment, are indicated by dashes. Single and double underlining indicates the short repeats that might be involved in deletions via slipped mispairing during DNA replication. Open boxes a1 and a2 indicate $\mathrm{B} 2$-homologous regions; $\mathrm{b} 1$ and $\mathrm{b} 2$ indicate $\mathrm{B} 3$-homologous region; and $\mathrm{c} 1, \mathrm{c} 2$ and $\mathrm{c} 3$ indicate regions homologous to the 1.9-kb plasmid-like DNA of Black Mexican Sweet maize. The nucleotide sequences of M1, M2 and M3 has been deposited in the DDBJ, EMBL, NCBI and GSDB databases under the accession number D50618, D50619 and D50620, respectively.

With respect to functional aspects of the sequences, only one open reading frame (ORF) of $399 \mathrm{bp}$ (beginning at position 2,430 and ending at position 2,032 on the opposite strand) was found in M1; two ORFs, of $309 \mathrm{bp}$ (beginning at 1,748 and ending at 2,056) and $264 \mathrm{bp}$ (beginning at 2,034 and ending at 1,771 on the opposite strand), respectively, were found in M2; and no ORF of longer than 200 bp was found in M3. B1 also has ORFs of $243 \mathrm{bp}$ and $288 \mathrm{bp}$, (Shikanai et al., 1987). These ORFs are not conserved in B1, M1 and M2 and, therefore, these ORFs seem not to encode any essential proteins.

Many short repeated sequences (less than $25 \mathrm{bp}$ ) were found, but no long repeat and stem and loop structure was found in the sequence of M1, M2 and M3, in contrast to the long direct or inverted repeat and hairpin structure, respectively, of the mitochondrial plasmid-like DNAs of Vicia faba (Wahleithner and Wolstenholme, 1987) and the pO plasmid of Beta vulgaris (Hansen and Macker, 1984).

\section{Comparison of sequences of $M 1, M 2, M 3$ and $B 1$}

We compared the sequences of M1, M2, M3 and B1 and diagrams of homologous sequences are shown in Figure 3. Hatched boxes indicate M1 or regions that are more than $90 \%$ homologous to M1; open boxes indicate the unique regions of B1 and M3 with no homology to M1. Over 95\% of the nucleotide sequence of M2 exhibits homology to M1, but M2 lacks 388 bp of M1 (beginning at 884 and ending at 1,271$)$. Incomplete repeats of $15 \mathrm{bp}$ of (T/A) GTTG (A/C) (A/C) TCATAAAA are present at the both borders of this deleted region in $\mathrm{M} 1$, and this element is present as one copy only in M2 in the corresponding region (indicated by double underlining in Figure 2 and by black arrowheads in Figures 3 and 4). About 90\% of the sequence of B1 is homologous to M1, and B1 lacks 323 bp of M1 (beginning at 871 and ending at 1,193). Small repeats of the ACC triplet are also present at both borders of this deleted region in M1 and this element is present as a single copy in B1 at the corresponding position (indicated by single underlining in Figure 


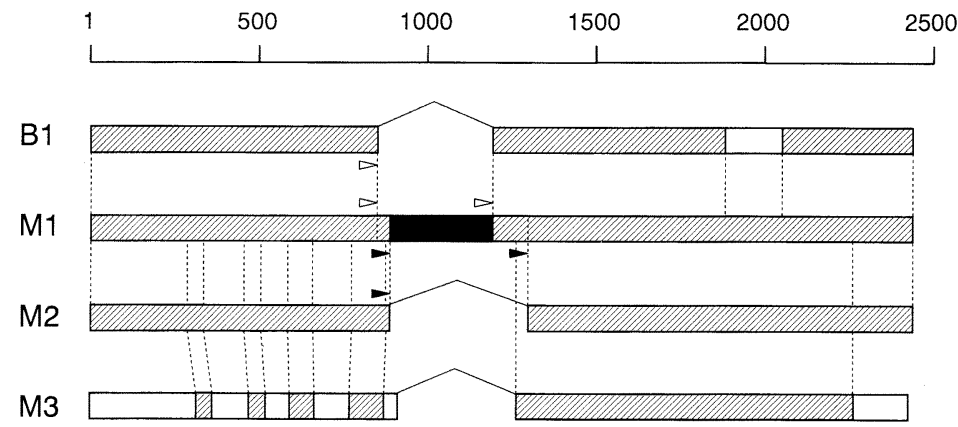

Fig. 3. A comparison of the sequences of members of the B1 family. Homologous and unique regions are indicated as boxes. The scale indicates nucleotide positions of M1 (see Figure 2). Hatched boxes indicate regions homologous to $\mathrm{M} 1$ and open boxes indicate the regions with no homology to M1. The unique region of M1 that is absent in B1, M2 and M3 is indicated as a closed box. White and black arrowheads indicate the small repeats of $3 \mathrm{bp}$ and $15 \mathrm{bp}$ that are indicated by single and double underlining in Figure 2.

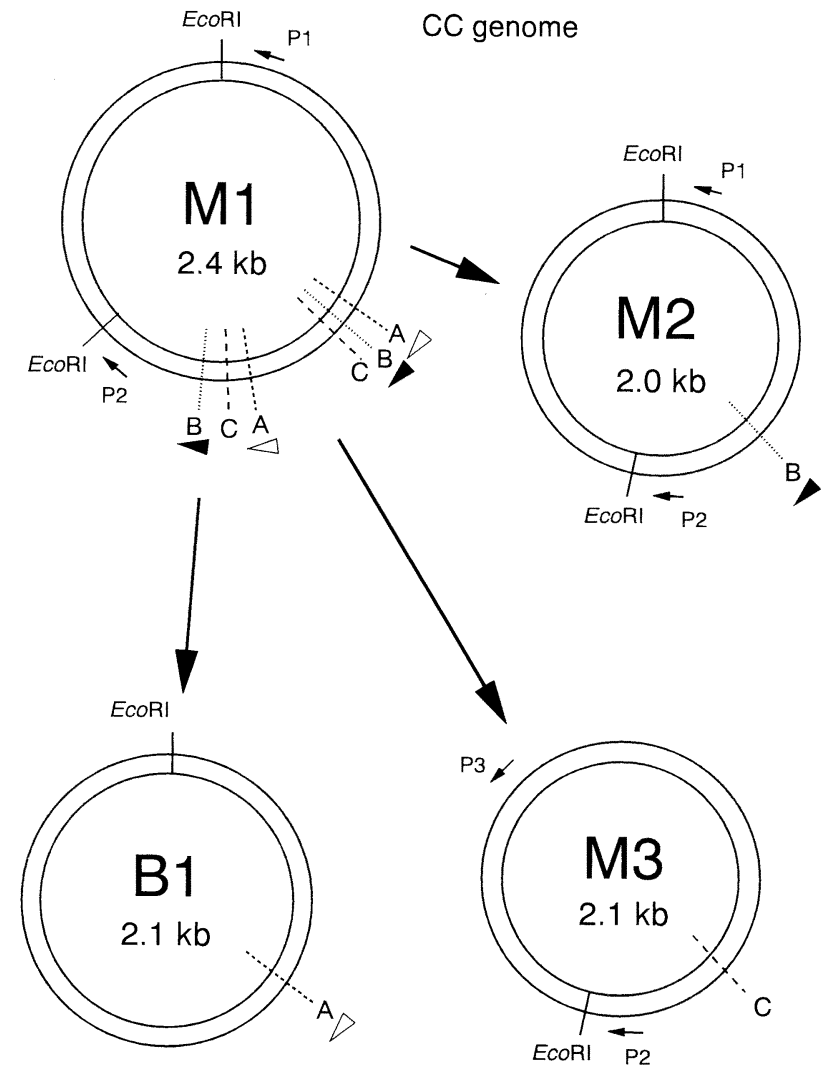

AA genome 
2 and white arrowheads in Figures 3 and 4). In contrast to B1 and M2, only about $60 \%$ of M3 is homologous to M1. M3 also lacks $343 \mathrm{bp}$ of M1 (beginning at 902 and ending at 1,244), but no repeat is found around this deleted region in M1 or at a corresponding site in M3. Thus, $291 \mathrm{bp}$ of the sequence of M1 is absent from B1, M2 and M3 (indicated by a filled box in Figure 3).

\section{DISCUSSION}

We cloned and sequenced these novel mitochondrial plasmid-like DNAs, namely, M1, M2 and M3, from strains in the genus Oryza with CC and CCDD genomes. A comparison of sequences revealed that these molecules have a highly conserved sequence over a wide region (Fig. 3). In B1, $322 \mathrm{bp}$ of $\mathrm{M} 1$, located between small triplet repeats, is deleted. In $\mathrm{M} 2$, as in $\mathrm{B} 1,388 \mathrm{bp}$ of $\mathrm{M} 1$, located between imperfect repeats of $15 \mathrm{bp}$, is deleted. In $\mathrm{B} 1$ and $\mathrm{M} 2$, these 3-bp or 15-bp repeats are present as single copies at the position that corresponds to the deleted region in M1. In M3, no similar repeats are found. From our present results, it is suggested that B1 and M2 arose from M1 by recombination or slipped mispairing during DNA replication. Therefore, we propose the following reasonable hypothesis about the generation and diversification of the B1 family in the genus Oryza (Fig. 4). First, an original plasmid-like DNA, probably similar to M1, was present in mitochondria of ancient species in the genus Oryza. During evolution, parts of this original plasmid-like DNA were deleted by intra-molecular recombination or by slipped mispairing during DNA replication, and prototypes of B1, M2 and M3 were generated. During further evolution and diversification of the genus Oryza, several mutations, such as deletions, insertions, or base substitutions, and recombination with other plasmid-like DNAs occurred and the B1 family was generated as a result. M3 exhibits less homology to M1 than B1 does and repeats are not retained in M3. Thus, M3 seems to have differentiated at the earliest stage of differentiation of B1 family. B1 also exhibits less homology to M1 than M2 does and retained repeats are only $3 \mathrm{bp}$ in length. Therefore, we suggest that the differentiation occurred in order M1 to M3 and then to B1 and M2.

Slipped mispairing between repeated sequences during DNA replication has been verified in vivo and in vitro as a mechanism that might be responsible for the generation of the polymorphic regions and large-scale deletions found in mam-

Fig. 4. The schematic representation of the explanation for the generation of members of the B1 family of mitochondrial plasmid-like DNAs during the diversification of the genus Oryza. Large arrows indicate the differentiation of the B1 family, as described in the text, and small arrows, labeled $\mathrm{P} 1, \mathrm{P} 2$ and P3, indicate primers 1, 2 and 3, respectively, that were used for amplification of partial sequences of M1, M2 and M3 by PCR. The dashed lines of A, B and C indicate the deleted regions of $\mathrm{M} 1$ and the corresponding sites in B1, M2 and M3, respectively. White and black arrowheads indicate the same sites as in Figure 3 (indicated as single and double underlining in Figure 2). 
malian mitochondrial genomes (Madsen et al., 1993). In mitochondria of higher plant, recombination across large and small repeated sequences is thought to provide an explanation for the large and complex genomes (for review, see André et al., 1992). Slipped mispairing during DNA replication, rather than intramolecule recombination, might result in the deletion of the parts of plasmid-like DNAs because the unique region of M1 that is deleted in B1, M2 and M3 seems to be too small to make the loop structures that are postulated to be intermediates during recombination.

To date, the genus Oryza has been studied on their relationships among the genome types of AA, BB, BBCC, CC, CCDD, EE and FF. Dally and Second (1990) and Second and Wang (1992) studied RFLPs of the chloroplast and mitochondrial DNAs in the genus Oryza, and they showed the phylogenetic relationships among each genome type regarding to cytoplasms. They also suggested that the results of their analyses are consistent with results of previous evaluations of genetic diversity and phylogeny based on isozymic or RFLP analyses of nuclear DNAs within the genus Oryza (Second, 1982, 1985; Glaszmann, 1987; Wang et al., 1992). From our present results, we suppose the presence of an original plasmid-like DNA which is similar to M1 in the ancient species of the genus Oryza before diversified into several genome types. The presence of this original plasmid-like DNA and our hypothesis of the generation of B1 family are consistent with previous studies about the phylogenetic relationships among the genome types in the genus Oryza.

The authors express their appreciation to A. Nakazono of The University of Tokyo for his valuable suggestions, and to A. Kanno of Tohoku University for his guidance during the experiments. This research was supported by Grants-in-Aid from the Ministry of Education, Science, Sports and Culture of Japan.

\section{REFERENCES}

André, C., Levy, A. and Walbot, V. (1992). Small repeated sequences and the structure of plant mitochondria. Trends Genet. 8, 128-132.

Chase, C. D. and Pring, D. R. (1985). Circular plasmid-like DNAs from mitochondria of Sorghum bicolor. Plant Mol. Biol. 5, 303-311.

Chase, C. D. and Pring, D. R. (1986). Properties of the linear N1 and N2 plasmid-like DNAs from mitochondria of cytoplasmic male-sterile Sorghum bicolor. Plant Mol. Biol. 6, 53-64.

Dally, A. and Second, G. (1990). Chloroplast DNA diversity in wild and cultivated species of rice (Genus Oryza, section Oryza). Cladistic-mutation and genetic-distance analysis. Theor. Appl. Genet. 80, 209-222.

Flamand, M. C., Duc, G., Goblet, J. P., Hong, L. and Louis, O. (1993). Variant mitochondrial plasmids of broad bean arose by recombination and are controlled by the nuclear genome. Nucleic Acids Res. 21, 5468-5473.

Glaszmann, J. C. (1987). Isozymes and classification of Asian rice varieties. Theor. Appl. Genet. 74, 21-30.

Hansen, B. M. and Macker, K. A. (1984). DNA sequence and transcription of a DNA minicircle 
isolated from male-fertile sugar beet mitochondria. Nucleic Acids Res. 12, 747-756.

Honda, H. and Hirai, A. (1990). A simple and efficient method for identification of hybrids using nonradioactive rDNA as probe. Jpn. J. Breed. 40, 339-348.

Iwahashi, M., Nakazono, M., Kanno, A., Sugiura, K., Ishibashi, T. and Hirai, A. (1992). Genetic and physical maps and clone bank of mitochondrial DNA from rice. Theor. Appl. Genet. 84, 275-279.

Kanazawa, A., Sakamoto, W., Nakagahra, M., Kadowaki, K., Tsutsumi, N. and Tano, S. (1992). Distribution and quantitative variation of mitochondrial plasmid-like DNAs in cultivated rice (Oryza sativa L.). Jpn. J. Genet. 67, $309-319$.

Kemble, R. J. and Bedbrook, J. R. (1980). Low molecular weight circular and linear DNA of a mitochondria from normal and male-sterile Zea mays cytoplasm. Nature 284, 565-566.

Madsen, C. S., Ghivizzani, S. C. and Hauswirth, W. W. (1993). In vivo and in vitro evidence for slipped mispairing in mammalian mitochondria. Proc. Natl. Acad. Sci. USA 90, 7671-7675.

Miyata, S., Kanazawa, A., Tsutsumi, N., Sano, Y. and Hirai, A. (1995). Polymorphic distribution and molecular diversification of mitochondrial plasmid-like DNAs in the genus Oryza. Jpn. J. Genet. 70, 601-614.

Pring, D. R. and Lonsdale, D. M. (1985). Molecular biology of higher plant mitochondrial DNA. Int. Rev. Cytol. 97, 1-46.

Sakamoto, W., Momose, M., Tsutsumi, N., Tano, S. and Yamaguchi, H. (1989). Analysis of homology of small plasmid-like mitochondrial DNAs in the different cytoplasmic male sterile strains in rice. Jpn. J. Genet. 64, 49-56.

Second, G. (1982). Origin of the genic diversity of cultivated rice (Oryza spp.): study of the polymorphism scored at 40 isozymic loci. Jpn. J. Genet. 57, 25-57.

Second, G. (1985). Evolutionary relationships in the Sativa group of Oryza based on isozyme data. Génét. Sél. Evol. (Paris) 17, 89-114.

Second, G. and Wang, Z. Y. (1992). Mitochondrial DNA RFLP in genus Oryza and cultivated rice. Genet. Resour. Crop Evol. 39, 125-140.

Shikanai, T. and Yamada, Y. (1988). Properties of the circular plasmid-like DNA, B4, from mitochondria of cytoplasmic male-sterile rice. Curr. Genet. 13, 441-443.

Shikanai, T., Yang, Z. Q. and Yamada, Y. (1987). Properties of the circular plasmid-like DNA B1 from mitochondria of cytoplasmic male-sterile rice. Plant Cell Physiol. 28, 1243-1251.

Shikanai, T., Yang, Z. Q. and Yamada, Y. (1989). Nucleotide sequence and molecular characterization of plasmid-like DNAs from mitochondria of cytoplasmic male-sterile rice. Curr. Genet. 15, 349-354.

Thomas, C. M. (1986). The nucleotide sequence and transcription of minicircular mitochondrial DNAs associated with male-fertile and cytoplasmic male-sterile lines of sugarbeet. Nucleic Acids Res. 14, 9353-9370.

Wahleithner, J. A. and Wolstenholme, D. R. (1987). Mitochondrial plasmid DNAs of broad bean: nucleotide sequences, complex secondary structures, and transcription. Curr. Genet. 12, 55-67.

Wang, Z. Q., Second, G. and Tanksley, S. D. (1992). Polymorphism and phylogenetic relationships among species in the genus Oryza as determined by analysis of nuclear RFLPs. Theor. Appl. Genet. 83, 565-581.

Yamaguchi, H. and Kakiuchi, H. (1983). Electrophoretic analysis of mitochondrial DNA from normal and male sterile cytoplasm in rice. Jpn. J. Genet. 58, 607-611. 